\title{
Velocity space resolved absolute measurement of fast ion losses induced by a tearing mode in the ASDEX Upgrade tokamak.
}

\author{
J.Galdon-Quiroga ${ }^{1,2}$, M.Garcia-Munoz ${ }^{1,2}$, \\ L.Sanchis-Sanchez $^{1,2}$, M.Mantsinen ${ }^{3,4}$, S.Fietz $^{5}$, \\ V.Igochine ${ }^{5}$, M.Maraschek $^{5}$, M.Rodriguez-Ramos ${ }^{1,2}$, \\ B.Sieglin ${ }^{5}$, A.Snicker ${ }^{5}$, G.Tardini ${ }^{5}$, D.Vezinet ${ }^{5}$, M. Weiland $^{5}$, \\ L.G.Eriksson ${ }^{6}$, the ASDEX Upgrade Team and the \\ EUROfusion MST1 Team $\ddagger$ \\ 1 Dept. of Atomic, Molecular and Nuclear Physics, University of Seville, Avda. \\ Reina Mercedes, 41012 Seville, Spain \\ 2 Centro Nacional de Aceleradores CNA (Universidad de Sevilla, Junta de \\ Andalucia, CSIC), Avda. Thomas A. Edison 7, 41092 Seville, Spain \\ 3 Barcelona Supercompunting Centre (BSC), Barcelona, Spain \\ 4 ICREA, Pg.Lluis Companys 23, 08010 Barcelona, Spain \\ 5 Max Planck Institute for Plasma Physics, Boltzmannstr. 2, 85748 Garching, \\ Germany \\ 6 European Commission, Directorate General for Research and Innov., B-1049 \\ Brussels, Belgium \\ E-mail: jgaldon@us.es \\ December 2017
}

\begin{abstract}
.
Absolute flux of fast ion losses induced by tearing modes have been measured by means of fast ion loss detectors (FILD) for the first time in RF heated plasmas in the ASDEX Upgrade tokamak. Up to $30 \mathrm{MW} / \mathrm{m}^{2}$ of fast ion losses are measured by FILD at $5 \mathrm{~cm}$ from the separatrix, consistent with infra-red camera measurements, with energies in the range of $250-500 \mathrm{keV}$ and pitch angles corresponding to large trapped orbits. A resonant interaction between the fast ions in the high energy tail of the ICRF distribution and a $m / n=5 / 4$ tearing mode leads to enhanced fast ion losses. Around $9.3 \pm 0.7 \%$ of the fast ion losses are found to be coherent with the mode and scale linearly with its amplitude, indicating the convective nature of the transport mechanism. Simulations have been carried out to estimate the contribution of the prompt losses. A good agreement is found between the simulated and the measured velocity space of the losses. The velocity space resonances that may be responsible for the enhanced fast ion losses are identified.
\end{abstract}

$\ddagger$ See the author list of H. Meyer et al., Nucl. Fusion 57102014 (2017). 


\section{Introduction}

In magnetically confined fusion devices, fast (suprathermal) ions generated by external sources such as neutral beam injection (NBI) and ion cyclotron resonance heating (ICRH) play an important role in the fusion power generation through key operational issues such as current drive and plasma heating efficiency $[1,2,3]$. This is only possible provided a good confinement of the fast ion population. Otherwise, the loss of fast ions to the vessel walls may cause, if sufficiently intense, irreversible damage to plasma facing components $[4,5]$, which is likely to be untolerable for next generation machines like ITER [6].

Therefore, understanding the mechanisms that can lead to an increased radial transport and eventual loss of fast ions is imperative. In particular, the interaction between the fast ion population and a large variety of magnetohydrodynamic (MHD) fluctuations such as neoclassical tearing modes [7, 8], fishbones [9] and Alfven waves [10, 11, 12, 13] among others, has been observed to provoke enhanced fast ion losses. A deeper insight of such fundamental mechanisms can be gained from direct measurement of fast ion losses with scintillator based fast ion loss detectors (FILD) [14] which are typically mounted on a probe and placed near the plasma edge. FILD detectors work as magnetic spectrometers making use of the tokamak magnetic field to disperse fast ions into a scintillator plate, providing time resolved velocity space measurements of fast ion losses.

We hereby report on the damage suffered by the FILD1 head probe due to fast ion losses in the presence of MHD activity in an ICRF heated discharge in the ASDEX Upgrade (AUG) tokamak. These measurements are made until the heat (and light) protection is damaged by the escaping ions. Infrared camera measurements are, however, available until the end of the discharge. The broad set of diagnostics available at AUG provides a comprehensive coverage of the problem. The absolutely calibrated FILD detectors provide velocity space resolved measurements of the absolute flux of fast ion losses in the presence of a tearing mode for the first time. The measured fast ion heat load, up to $30 \mathrm{MW} / \mathrm{m}^{2}$, is consistent with infra-red camera measurements. The fast ion orbits measured by FILD overlap with the spatial structure of the mode, which is reconstructed by means of electron cyclotron emission (ECE) and soft X-ray (SXR) tomography measurements. Around $9.3 \pm 0.7 \%$ of the fast ion losses are found to be coherent with the $m / n=5 / 4$ tearing mode, corresponding to $3 \mathrm{MW} / \mathrm{m}^{2}$ approximately.

The paper is organised as follows: in section 2 an overview of the discharge is given and the main experimental observations are presented. In section 3
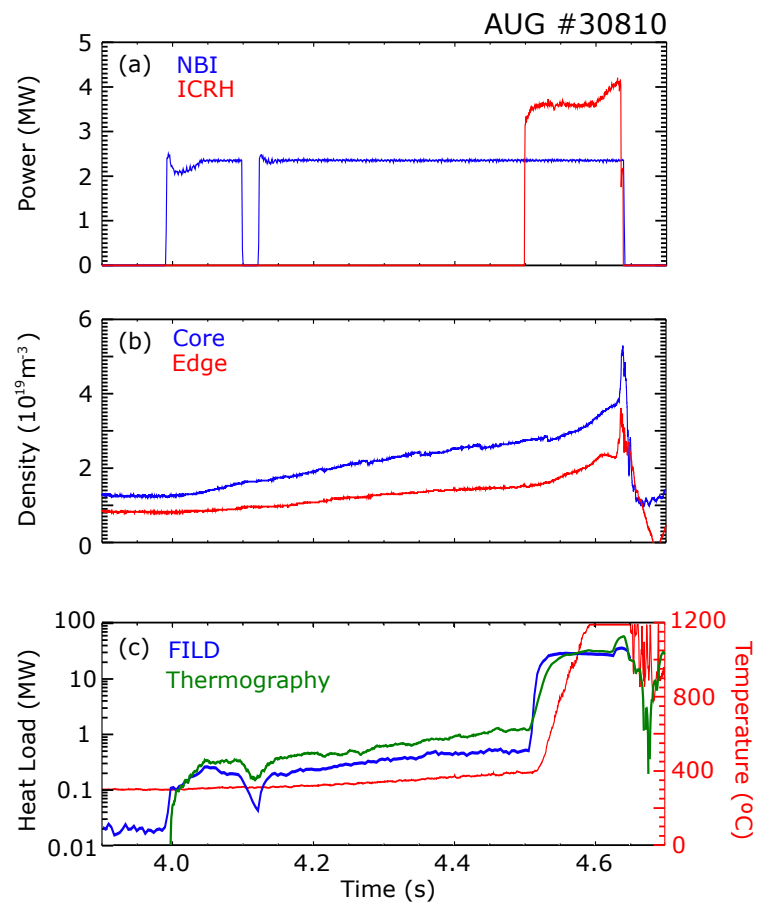

Figure 1. Timetraces of the of AUG shot \#30810 in the time window of interest. (a) NBI power is shown in blue and ICRF power is shown in red. (b) Line integrated density in the core (blue) and in the edge (red). (c) Fast ion heat load measured with FILD1 is plotted in blue. The heat load measured by the thermography is plotted in green. The temperature in the head probe is plotted in red

the modelling goals and results are presented. Finally, in section 4 the conclusions are discussed.

\section{Loss of ICRF accelerated fast ions}

The experiment discussed here has been performed in the ASDEX Upgrade tokamak with a plasma current $I_{p}=1 \mathrm{MA}$ and a magnetic field $B_{t}=2.36 \mathrm{~T}$. $2.5 \mathrm{MW}$ of NBI heating with a main injection energy of $60 \mathrm{keV}$ are applied in three different phases: 0.3-1.0 s, 2.0-3.0 and $4.0-4.7$ s. $4 \mathrm{MW}$ of ICRF-heating are applied at $4.5 \mathrm{~s}$ with a frequency of $30 \mathrm{MHz}$ corresponding to the hydrogen minority heating and deuterium second harmonic heating. The hydrogen content in the deuterium plasma is around $5 \%$ as estimated by neutral particle analyzer (NPA) measurements. In Fig.1 an overview of the relevant phase of discharge is shown. The plasma density increases up to $3 \cdot 10^{+19} \mathrm{~m}^{-3}$ when the NBI is turned on at $4.0 \mathrm{~s}$. When the ICRH is switched on at $4.5 \mathrm{~s}$ the FILD1 signal increases dramatically in a time window of $100 \mathrm{~ms}$. The temperature in the FILD1 head probe, placed at $5 \mathrm{~cm}$ from the separatrix, increases up to $1200^{\circ} \mathrm{C}$ and is then damaged leading to the discharge termination due to radiative collapse.

The FILD diagnostic [14] is a scintillator based 
fast-ion loss detector which is mounted on a probe and placed in the far scrape-off layer. In AUG several FILD detectors are installed at different toroidal and poloidal positions. In this work we focus on the measurements provided by the FILD1 detector located near the ASDEX Upgrade midplane at $z=0.3$ $m$. The probe consists mainly on a scintillator, a collimator and a graphite head protection with a tungsten coating. Fast ion orbits that reach the probe head are collimated before striking on the scintillator. The light yielded by the scintillator is collected by an acquisition system consisting of two branches: one, a set of photomultiplier tubes (PMTs) covering different velocity space regions of the losses, which provides a time resolution of $1 \mathrm{MHz}$; the other, a fast CMOS camera imaging the scintillator which provides a good spatial resolution for the identification of the velocity space of the losses with a time resolution of $1 \mathrm{~ms}$.

An infra-red camera with a frame rate of $2 \mathrm{kHz}$ measures the heat flux on different points of the FILD1 head (Fig.2) [15]. The temporal evolution of the heat load on the detector head at the approximate position of the collimator pinhole is shown in Fig.3. Before the ICRH phase the heat load stays below $5 \mathrm{MW} / \mathrm{m}^{2}$. As soon as the ICRH is switched on, the heat load increases peaking at around $30 \mathrm{MW} / \mathrm{m}^{2}$. At $4.63 \mathrm{~s}$ the FILD1 head probe is damaged as illustrated in Fig.2. A dramatic increase of the FILD1 signal is observed in Fig.3 (c).

The evolution of the velocity space of the absolute flux of fast-ion losses as measured by the FILD1 CMOS camera is shown in Fig.4. Fig.4 (a) shows the measurement shortly before the ICRH phase. A spot corresponding to particles with energy $E=60 \mathrm{keV}$ and pitch angle $\Lambda=\cos ^{-1}\left(v_{\|} / v\right) \approx 60^{\circ}$ is identified as NBI prompt losses. In Fig.4 (b) the fast ion losses corresponding to ICRF heated ions start to be measured at larger gyroradii around $13 \mathrm{~ms}$ after the ICRH is turned on, with the spot centered at around $r_{L}=6 \mathrm{~cm}$. These correspond to energies of around 500 $\mathrm{keV}$ in the case of hydrogen ions and $250 \mathrm{keV}$ in the case of deuterium ions. In Fig.4 (c), $33 \mathrm{~ms}$ after the ICRH is turned on, the velocity space distribution has evolved and the fast-ion losses have increased.

The absolute calibration of the FILD systems in AUG allows the estimation of the fast-ion flux at the collimator pinhole [16]. The full characterization of the scintillating material together with the calibration of the FILD1 light acquisition system permits to estimate the flux of ions striking in the scintillator plate based on the CMOS camera measurements. The collimator factor, this is, the fraction of ions that are blocked by the collimator is calculated using the FILDSIM code. This way, the absolute flux of fast-ions in the FILD1 pinhole can be calculated as $\Gamma_{\text {pinhole }}=$
$\Gamma_{\text {scintillator }} / f_{\text {col }}$.

The fast-ion heat load measured by FILD1 (red and blue) is consistent with the infrared camera measurements (black) as shown in Fig.3 (c), indicating that the heat load is mostly coming from fast ion losses. The signal corresponding to two PMT channels (green and yellow) are plotted. The timetraces of these two signals are slightly different since they correspond to different regions of the velocity space of the losses. The FILD1 fast-ion heat load is obtained by integrating the CMOS camera signal in the whole velocity space of the losses. For $t>4.53$ some pixels of the CMOS camera in the region of interest saturate and therefore the fastion heat load measured by FILD consitutes only a lower boundary.

The FILD diagnostic by itself is not able to distinguish between different ion species. For this reason the fast-ion heat load measured with FILD1 has been evaluated assuming both, hydrogen (red) and deuterium (blue) ions. The estimated fast-ion heat load is similar in both cases, it is only around $20 \%$ smaller in the case of hydrogen ions. The reason for not having a large discrepancy is that the difference in the measured energy of both species is compensated by the difference in the light yield of the scintillator. In other words, the fast-ion heat load measured with FILD can be expressed as

$P_{F I L D} \propto \Gamma_{\text {scint }} \frac{E}{\xi}$

where $\Gamma_{\text {scint }}$ is the photon flux emmited by the scintillator, $E$ is the energy of the ions and $\xi$ is the scintillator yield, this is, the number of photons emmitted per incident ion, which is a function of the particle energy and species. Despite the energy of the measured hydrogen $(500 \mathrm{keV})$ is larger than the energy of the measured deuterium $(250 \mathrm{keV})$, the scintillator yield for hydrogen at $500 \mathrm{keV}$ is also larger than the scintillator yield for deuterium at $250 \mathrm{keV}$.

It can be noticed that the FILD1 signal increases faster than the IR camera signal. This can be explained in terms of the difference in response times for both diagnostics. The IR camera measurement is based on the temperature increase of the FILD1 head probe, which is naturally subject to some thermal inertia. On the other side, the FILD measurement is based on the scintillator light emission, which is a process with characteristic times in the order of $10^{2} \mathrm{~ns}$.

\subsection{Fast ion losses induced by MHD activity}

An intense MHD activity in the plasma is observed in the spectrogram obtained with magnetic pick-up coils (Fig.3(a)). As soon as the ICRF is switched on at $4.5 \mathrm{~s}$ fishbone activity is observed, leaving its characteristic footprint in the spectrogram as a frequency chirp at 


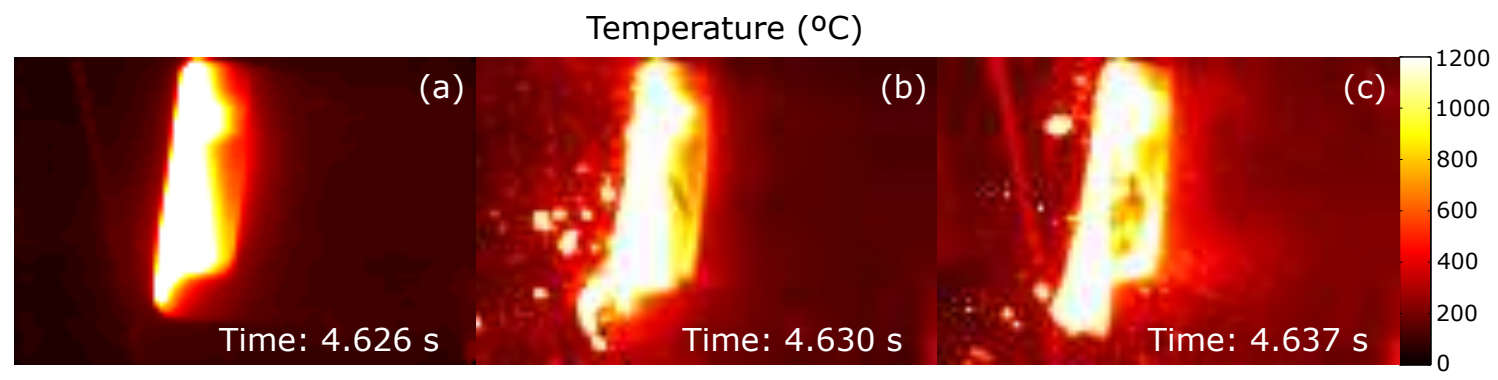

Figure 2. Infrarred camera frames showing the FILD1 head temperature at $t=4.626 \mathrm{~s}$ (a) just before the FILD1 head probe is damaged, and at $t=4.630 \mathrm{~s}$ (b) and $t=637 \mathrm{~s}$ (c) just after it.
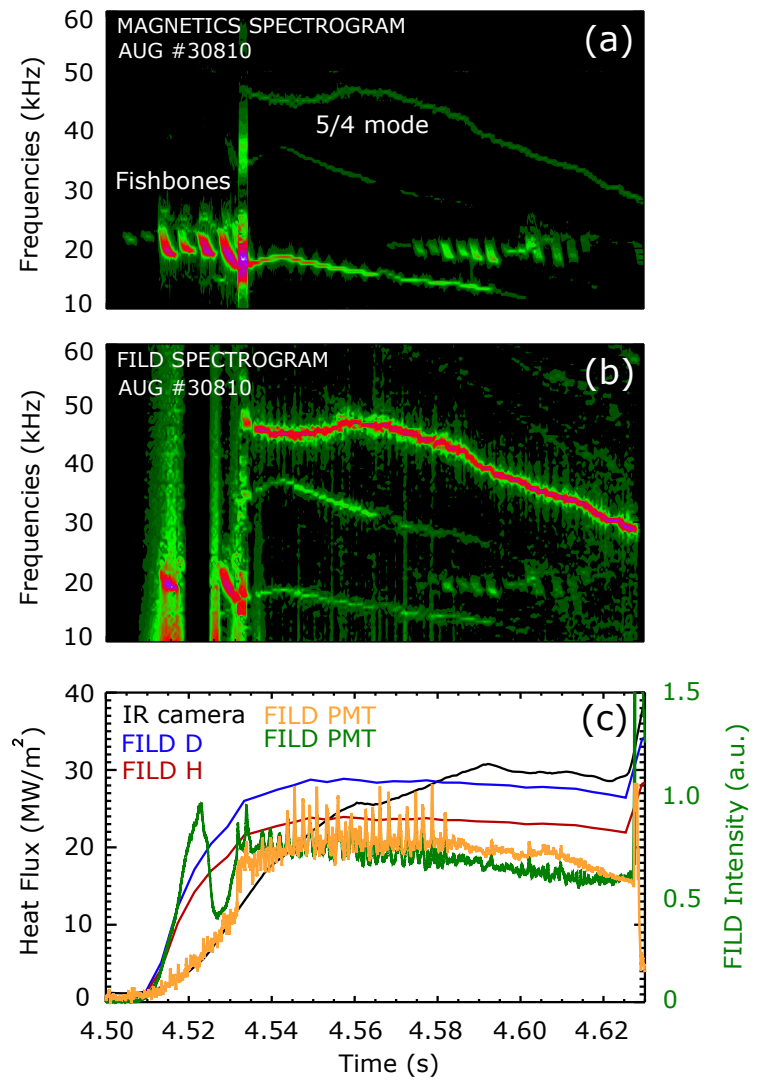

Figure 3. (a) Spectrogram performed to a magnetic pick-up signal at the time window corresponding to the ICRH phase. The spectrogram reveals the MHD activity in the plasma. (b) Spectrogram performed to the FILD1 signal taken from a PMT. A clear correlation is observed with the MHD activity measured by the magnetic signal. (c) Time trace of the heat flux measured by the infrared camera (black), the fast-ion heat flux measured by the FILD1 detector from the CMOS camera signal assuming hydrogen ions (blue) and deuterium ions (red) and the FILD1 signal of two different photomultiplier channels (green and yellow). around $20 \mathrm{kHz}$. At $4.53 \mathrm{~s}$ a sawtooth crash takes place which triggers several internal modes at a frequency of $45 \mathrm{kHz}, 35 \mathrm{kHz}$ and $20 \mathrm{kHz}$ approximately. This kind of event has been already reported in AUG[17]. At $4.58 \mathrm{~s}$ fishbone activity is again observed. Looking at the FILD spectrogram in Fig. 3(b) we can see a clear correlation with the MHD activity present in the plasma. This is indicative of an interaction between the MHD and the fast-ion population, leading to enhanced fast-ion radial transport and loss. The mode contributing most intensely to the fast-ion losses is the one starting with a frequency of $45 \mathrm{kHz}$ and sweeping down to $33 \mathrm{kHz}$ at $4.62 \mathrm{~s}$. This mode is found to be a tearing type by means of the 1D standard electron cyclotron emission (ECE) diagnostic available in AUG, which consists of a 60 channels heterodyne radiometer with a sampling rate of $1 \mathrm{MHz}$ and a spatial resolution of $\sim 12 \mathrm{~mm}$ [18]. The method described in [17] allows to discern between kink and tearing type modes by identifying phase shifts in the timetrace of the ECE signal measuring at the resonant surface. The poloidal and toroidal mode numbers have been identified as $m=5$ and $n=4$ respectively by means of the magnetic pick-up coils and the soft Xray (SXR) diagnostic. The perturbation is then lying near the $\mathrm{q}=1.25$ surface which is in agreement with the 2D-tomographic SXR reconstruction of the mode, as shown in Fig.5. On the other hand, the mode starting at a frequency of $\sim 20 \mathrm{kHz}$ and sweeping down to $\sim 15$ $\mathrm{kHz}$ is found to be a core $m / n=1 / 1$ mode by means of the magnetic pick-up coils and also in agreement with the SXR tomography, while the other coherent mode, sweeping in frequency from $\sim 35$ to $\sim 30 \mathrm{kHz}$ is a higher harmonic of the latter one.

The proposed mechanism underlying the enhanced fast-ion losses is a resonant interaction between the MHD and the fast particles. Particles fulfilling the resonant condition $[19,20] \Omega_{n, p}=n \omega_{\phi}-p \omega_{\theta}-\omega_{M H D}=0$ may experience an exchange of momentum and energy with the wave. In the equation $n$ is the toroidal mode number, $p$ the poloidal harmonic, $\omega_{\phi}$ the toroidal precession frequency, $\omega_{\theta}$ the poloidal bounce frequency 


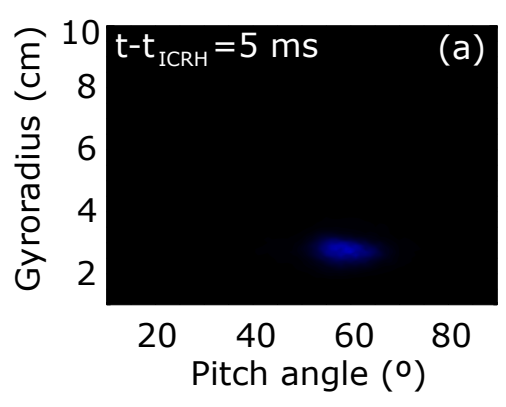

FILD intensity (ions/s/cm/o)

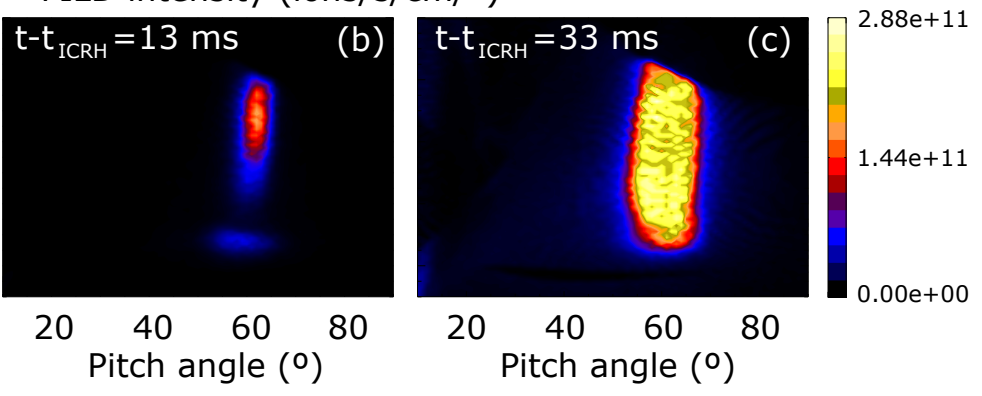

Figure 4. Time evolution of the velocity space resolved absolute flux of fast ion losses in the scintillator measured with FILD1 (i.e: without including the collimator factor).(a) Shows the velocity space $5 \mathrm{~ms}$ prior to the ICRH phase. A spot corresponding to the NBI prompt losses at $r_{L}=3.0 \mathrm{~cm}$ and $\Lambda=60^{\circ}$ is observed.(b) Shows the velocity space $13 \mathrm{~ms}$ after the ICRH is turned on. A new spot corresponding to ICRH accelerated fast ions is observed with Larmor radius around $6 \mathrm{~cm}$ and $\Lambda=60^{\circ}$. (c) Shows the velocity space $33 \mathrm{~ms}$ after the ICRH is turned on. The spot corresponding to ICRF accelerated ions has fully developed.
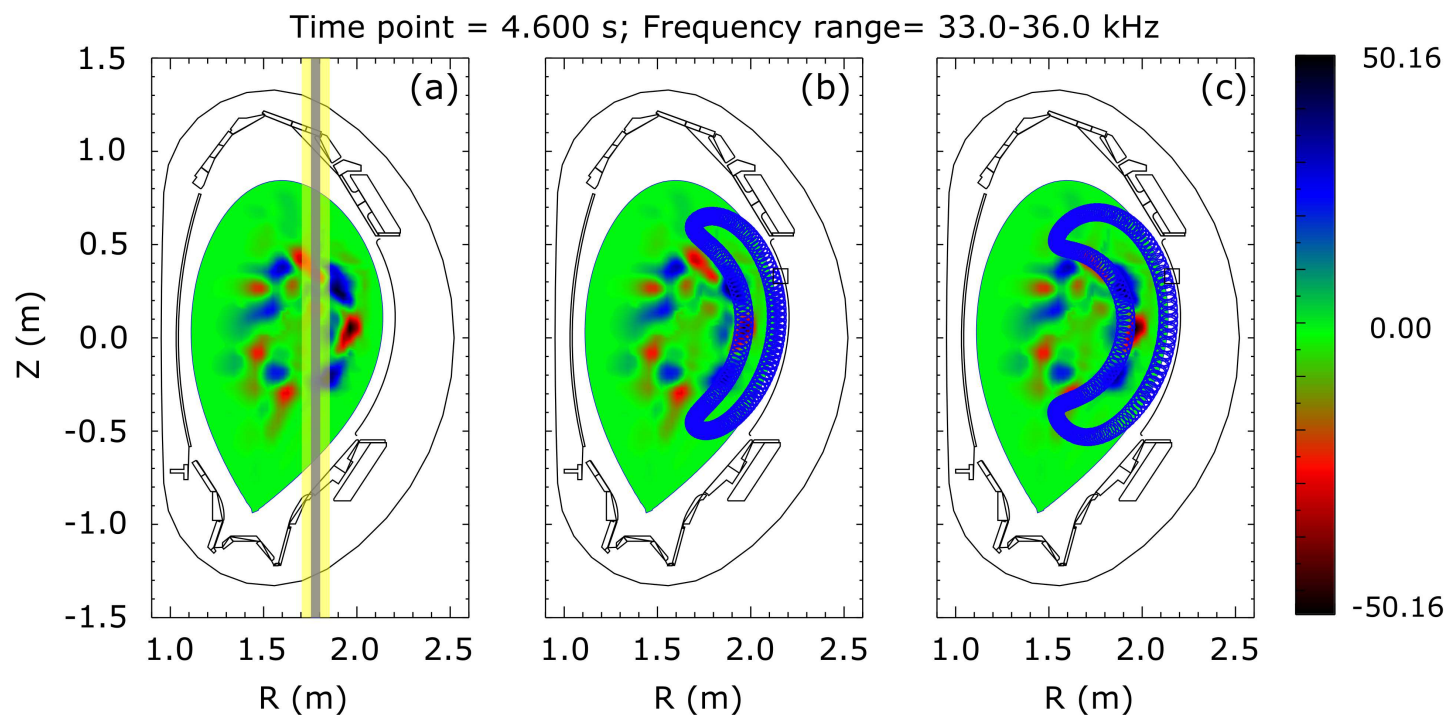

Figure 5. Poloidal view showing the tomographic reconstruction of the $5 / 4$ tearing mode by means of the SXR diagnostic. In (a) the ICRF resonance layers for hydrogen and deuterium are overlayed. In (b) an orbit corresponding to a deuterium ion at $250 \mathrm{keV}$ backtraced from the FILD1 detector is shown. In (c), the same for a hydrogen particle at $500 \mathrm{keV}$. It can be noticed that both trajectories overlap with the spatial structure of the mode.

and $\omega_{M H D}$ the MHD mode frequency, where all the frequencies are considered in the laboratory frame. A more detailed analysis of the FILD1 photomultiplier signal imaging the region corresponding to the ICRF losses reveals a linear dependence between the intensity of the coherent fast-ion losses and the magnetic perturbation at the corresponding mode frequencies, shown in Fig.6. This is consistent with the fact that the waveparticle momentum exchange in the resonance is proportional to the perturbation amplitude $\delta B$, indicating the convective nature of this transport mechanism $[20,21]$. The ratio between the amplitude of the FILD signal at the mode frequency and the total signal shows that the convective losses due to the $5 / 4$ tearing mode constitute $9.3 \pm 0.7 \%$ of the measured fast-ion losses, which corresponds to approximately $3 \mathrm{MW} / \mathrm{m}^{2}$. The other two coherent modes contribute to the total FILD signal by less than $2 \%$. However, in addition to the coherent fast ion losses, an overlapping of resonances in the particles phase space can lead to increased fast ion losses via stochastic diffusion [20]. Given the presence of multiple MHD modes and the size of the fast ion orbits shown in Fig.5, it is likely that the ICRH accelerated ions interact simultaneously with the multiple MHD modes, thus leading to orbit stochasticity and diffusion. Finite Larmor Radious (FLR) effects are, however, not discussed in the paper as they would require expensive simulations that are beyond the scope of this paper. These stochastic losses appear in the FILD signal as an incoherent component which cannot be directly distinguished from the prompt fast-ion losses. An estimation for the latter can be provided by 


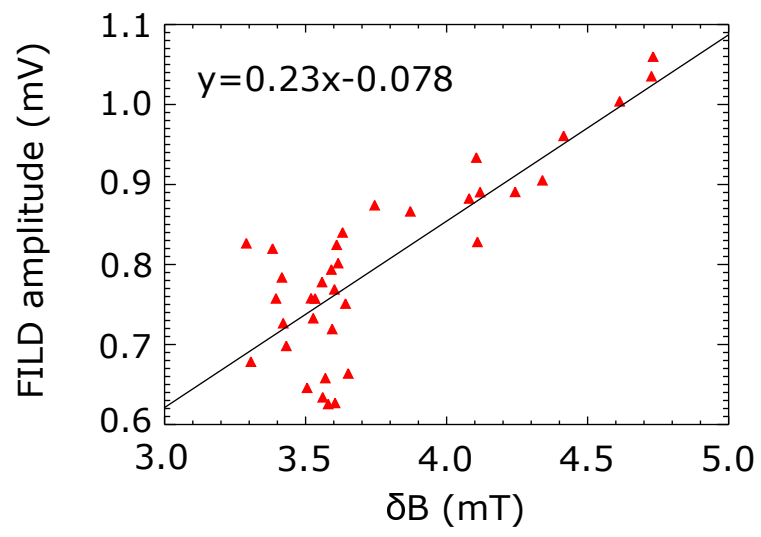

Figure 6. Amplitude of the FILD1 signal against the amplitude of the magnetics signal at the frequency corresponding to the $5 / 4$ tearing mode. A linear correlation is observed.

modelling.

\section{Modelling}

In order to model the fast ion losses in the presence of ICRF heating, we have first calculated the power coupled to the plasma using the PION code [22]. The power split obtained with PION shows that around $73 \%$ of the coupled power corresponds to hydrogen damping, $20 \%$ to second-harmonic deuterium damping and $7 \%$ to direct electron damping. This is in agreement with the ICRF coupled power calculated with TRANSP-TORIC as shown in Fig.7. To build the fast-ion distribution function, PION introduces a simple model [23] which reproduces the "rabbit-ear" feature typical for ICRH fast-ion distributions [24]:

$$
\begin{aligned}
& f_{0}(v, \xi, \psi)=F(v, \psi) \cdot C(v, \psi) \cdot \\
& \left\{\exp \left[-\left(\frac{\xi-\xi_{R}(\psi)}{\Delta \xi(v, \psi)}\right)^{2}\right]+\exp \left[-\left(\frac{\xi+\xi_{R}(\psi)}{\Delta \xi(v, \psi)}\right)^{2}\right]\right\}
\end{aligned}
$$

where $v$ is the particle velocity, $\xi$ is the pitch angle of the particle orbit in the outer midplane and $\psi$ is the poloidal magnetic flux function. $F(v, \psi)$ is the pitch angle averaged distribution function, $C(v, \psi)$ is a normalisation constant such that the flux surface averaged density is consistent with the pitch angle averaged distribution, $\xi_{R}$ is the pitch angle in the outer midplane of a particle whom turning points are at the resonance layer, and $\Delta \xi$ is a parameter that controls the width of the "rabbit ears". In order to estimate $\Delta \xi$ we assume that:

$\eta_{\text {eff }}^{2}(v)=\frac{1}{2} \int \eta^{2} f_{0}\left(v, \xi\left(\eta, \psi, \theta_{R}\right), \psi\right) d \eta$

where $\eta$ is the pitch angle of the particle evaluated at the resonance point, meaning that $\theta_{R}$ corresponds to the position where $\omega=n \omega_{c i}\left(\psi, \theta_{R}\right)$. As a rough approximation we take $\eta_{e f f}^{2}$ to have the same functional form as $\mu_{e f f}^{2}$ in Ref.[22].

The energy spectra for both, hydrogen and deuterium species calculated with PION is shown in Fig.8. It can be seen that the amplitude of the high energy tail is larger for hydrogen ions than for deuterium ions.Since most of the power is coupled to the hydrogen ions, we will focus on these in the modelling of the fast ion losses.

Provided the hydrogen fast-ion distribution calculated with PION, the orbit following code ASCOT [25] has been used to calculate the contribution to the fastion heat load in the FILD head probe due to prompt losses, as well as the velocity space of these. The computation of the fast-ion heat load with ASCOT has been validated for NBI prompt losses in previous works[16]. ASCOT includes a realistic 3D model for the vessel wall and the geometry of the FILD detector. In this case the simulations were carried out in full-orbit and $10^{6}$ Monte Carlo markers were followed during $1 \mathrm{~ms}$. The toroidal field ripple was included in the magnetic equilibrium, but no internal MHD perturbation was considered in the simulation.

For a direct comparison with the experimental measurements, a synthetic FILD signal is built by applying the FILDSIM code to the velocity space distribution of the losses given by ASCOT in the FILD1 head. FILDSIM reproduces the fast-ion distribution pattern measured in the scintillator using a weighting function that accounts for the collimator factor and the finite resolution of the detector in energy and pitch angle. The velocity space distribution of the ions reaching the FILD1 head simulated with ASCOT is shown in Fig.9 (a), while Fig.9 (b) shows the synthetic FILD1 signal after applying the FILDSIM model. The difference in the amplitude of the velocity space fast ion distribution between Figs.9 (a) and (b) is due to the effect of the collimator factor, which takes into account the ratio of particles that are blocked by the 3D collimator geometry. The simulated velocity space of the losses is in fairly good agreement with the FILD1 measurement (Fig.4 (c)). It can be observed that the calculated ASCOT distribution includes a set of ions with low energies $\left(\rho_{L}<2 \mathrm{~cm}\right.$ and $60^{\circ}<$ $\Lambda<80^{\circ}$ ) which are not measured by FILD. This is reproduced by the synthetic signal since these particles are blocked by the collimator due to their small Larmor radius. On the other hand, the heat-load in the FILD1 head probe obtained with ASCOT due to prompt fast ion losses including the effect of the toroidal field ripple is around $2.5 \mathrm{MW} / \mathrm{m}^{2}$. This is well below the total heat load measured experimentally. As discussed in Sec.2, it should be noticed that the total fast ion 
(a)

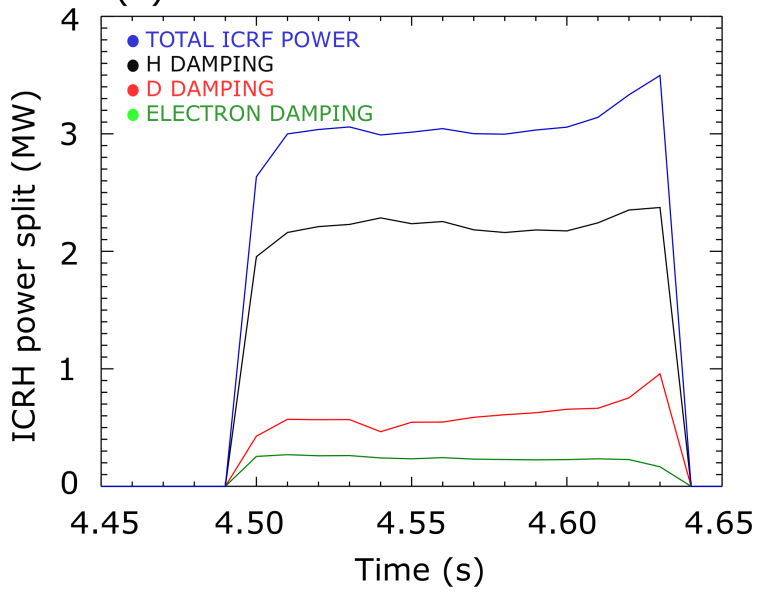

(b)

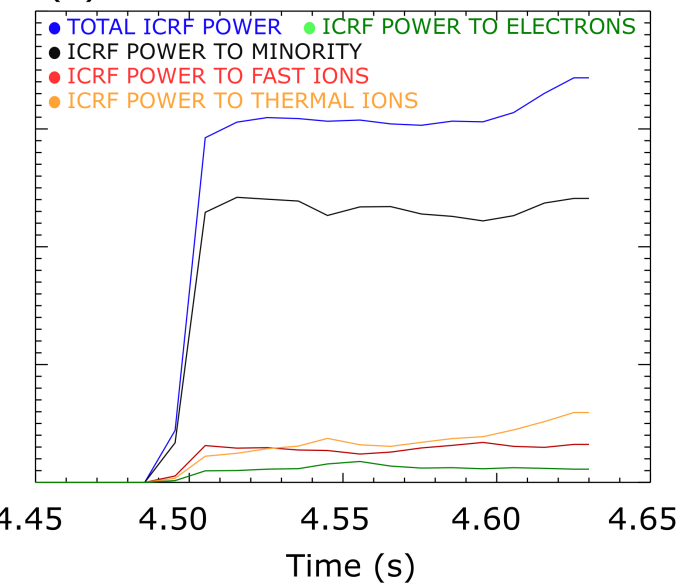

Figure 7. ICRF power coupled to the different species present in the plasma simulated with PION (a) and with TRANSP-TORIC (b). The results given by both codes are in good agreement. Most of the power (73\%) is transferred to hydrogen ions, $20 \%$ is transferred to deuterium via second harmonic heating, and around $7 \%$ is transferred to electrons. In the PION simulation (a), the power transferred to deuterium ions is indicated in red. This includes both, the power transferred to thermal deuterium and to fast deuterium ions, which are present in the plasma due to neutral beam injection. In the TRANSP simulation (b), the power tranferred to deuterium is explicitely separated into thermal species (orange) and fast ions (red).

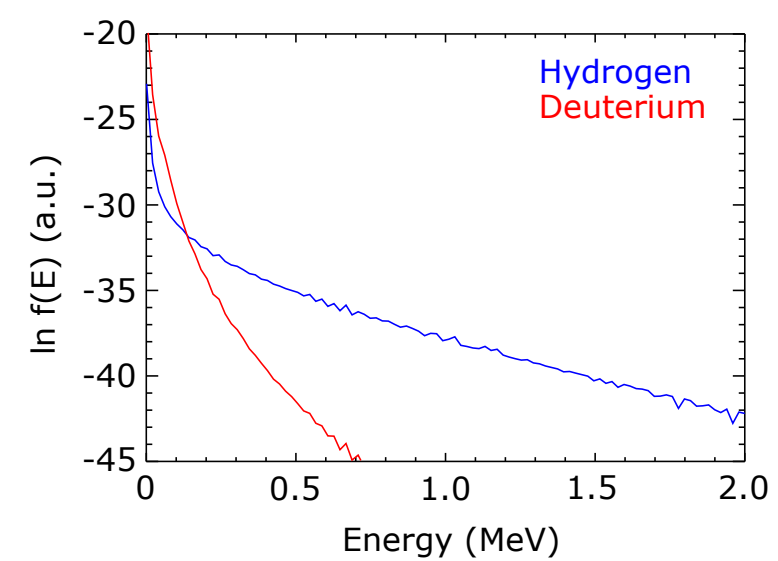

Figure 8. Energy spectrum of ICRF accelerated hydrogen (blue) and deuterium (red) calculated with PION.

heat load is a sum of different contributions: fastion prompt losses, convective losses due to a direct interaction between the fast-ions and individual MHD modes and diffusive losses due to the overlapping of phase space resonances in the presence of single or multiple MHD modes. The ASCOT simulation gives an estimate of the first of these terms, therefore highlighting the importance of the convective and diffusive losses induced by the MHD activity.

The velocity space of the resonances between fast ions passing through the FILD1 detector and the $5 / 4$ mode have been calculated by following orbits backwards in time started at the FILD1 detector position. The resonance lines are shown in Fig.10,

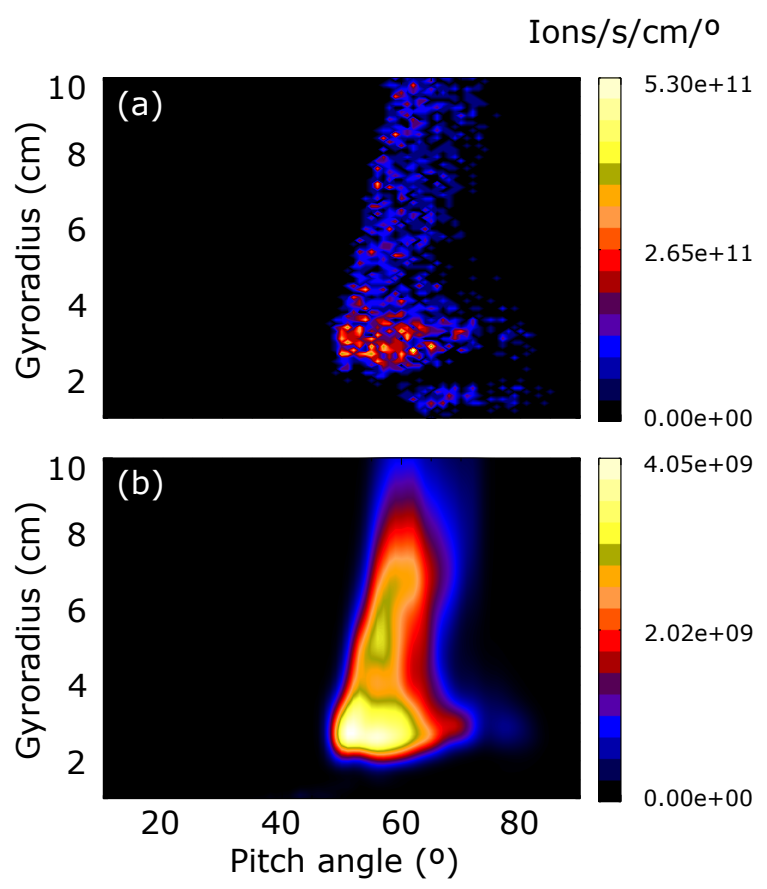

Figure 9. Simulated velocity space of the losses in the FILD1 detector. (a) Fast-ion distribution in the FILD1 head calculated with ASCOT using as input an ICRF distribution calculated with PION. (b) Synthetic FILD1 signal obtained after the application of the FILDSIM code. The result is in good qualitative agreement with the experimental measurement. 


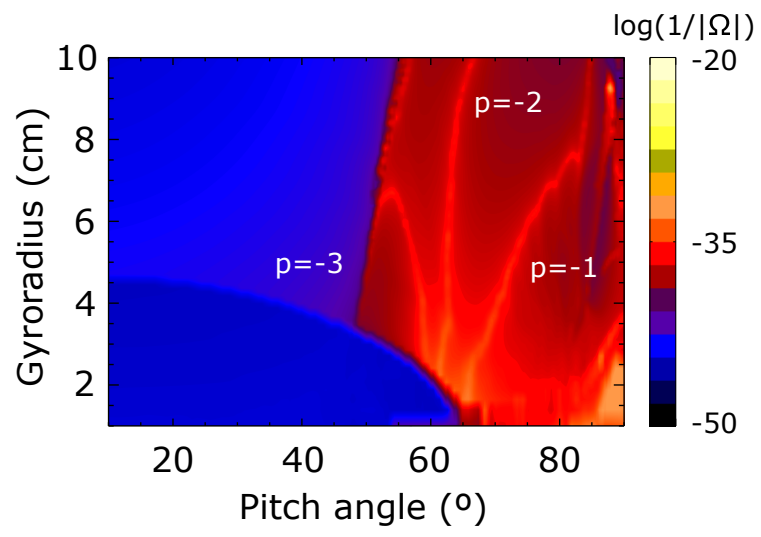

Figure 10. Velocity space of the resonances for fast-ion orbits at the FILD1 detector position. The different resonance lines correspond to different values of the poloidal harmonic $p$.

where the pitch angle and the gyroraius are evaluated at the FILD1 position. These represent the orbits fulfilling the resonant condition $\Omega_{n, p}=n \omega_{\phi}-p \omega_{\theta}-$ $\omega_{M H D}=0$. The different branches observed in the figure correspond to different values of the poloidal harmonic $p$. The resonance line associated to $p=-2$ overlaps with the FILD1 measurement.

The calculation has been performed for both, hydrogen and deuterium ions. The resonance lines are different for both species if plotted in the energy-pitch angle space. However, as it has been stated before, the FILD detector does not measure the energy directly but the Larmor radius of the particle. Due to the mass difference between both species, the resonance lines overlap when mapping them from energy to Larmor radius, given the magnetic field at the FILD position.

\section{Conclusions}

An unusually large amount of ICRF accelerated fast ion losses have been observed to damage the FILD1 head probe in the presence of MHD activity in the ASDEX Upgrade tokamak. The velocity space resolved fast ion heat load measured by the absolutely calibrated FILD1 detector is in good agreement with infra-red camera measurement, in the order of 30 $M W / m^{2}$. These fast ion losses are clearly correlated with MHD activity in the plasma.

The total heat load measured with FILD1 is the sum of different contributions: prompt losses, convective losses and diffusive losses. The fraction of the total fast ion heat load due to prompt losses is estimated to be around $2.5 \mathrm{MW} / \mathrm{m}^{2}$ by ASCOT simulations. The velocity space of the simulated losses is in fairly good agreement with the experimental measurement. The modelling results suggest that the remaining heat load could correspond to convective and diffussive fast ion losses induced by the MHD activity in the plasma. The mode contributing more intensely to the MHD induced fast ion losses is a $m / n=5 / 4$ tearing mode. Around $9.3 \%$ of the losses, corresponding to around $3 \mathrm{MW} / \mathrm{m}^{2}$, are coherent with the mode frequency and scales linearly with the mode amplitude, indicating the convective nature of this transport mechanism. The possible resonances responsible for these enhanced fast ion losses have been identified.

The experimental observations reveal the potential of MHD activity to reduce the confinement of the fast ion population and eventually provoke irreversible damage to plasma facing components.

\section{Acknowledgments}

This work has been carried out within the framework of the EUROfusion Consortium and has received funding from the Euratom research and training programme 2014-2018 under grant agreement No 633053. The views and opinions expressed herein do not necessarily reflect those of the European Commission. This research was supported in part by the Spanish Ministry of Economy and Competitiveness (Grant Nos. RYC2011-09152, FIS2015-69362-P, and ENE2012- 31087).

\section{References}

[1] W. W. Heidbrink and G.J. Sadler. Nucl. Fusion, 34:535, 1994.

[2] S.D. Pinches et al. Plasma Phys. Control. Fusion, 46:B187, 2004.

[3] A. Fasoli et al. Progress in the iter physics basis chapter 5: Physics of energetic ions. Nucl. Fusion, 47:S264, 2007.

[4] H.H. Duong et al. Nucl. Fusion, 33:749, 1993.

[5] R.B. White et al. Phys. Plasmas, 2:2871, 1995.

[6] M. Shimada et al. Progress in the iter physics basis chapter 1: Overview and summary 2007. Nucl. Fusion, 47:S1, 2007.

[7] M. Garcia-Munoz et al. Nucl. Fusion, 47:L10-L15, 2007.

[8] E. Poli et al. Phys. Plasmas, 15:032501, 2008.

[9] C. Perez von Thun et al. Nucl. Fusion, 50:084009, 2010.

[10] S.D. Pinches et al. Nucl. Fusion, 46:S904, 2006.

[11] M. Garcia-Munoz et al. Nucl. Fusion, 49:085014, 2009.

[12] M. Garcia-Munoz et al. Nucl. Fusion, 51:103013, 2011.

[13] M. A. Van Zeeland et al. Phys. of Plasmas, 18:056114, 2011.

[14] M. Garcia-Munoz et al. Rev. Sci. Instr., 80:053503, 2009.

[15] B. Sieglin. PhD thesis, Technical university Munich, 2014.

[16] M. Rodriguez-Ramos et al. Plasma Phys. Control. Fusion, 59:105009, 2017.

[17] V. Igochine et al. Phys. Plasmas, 21:110702, 2014.

[18] M. Willensdorfer et al. Plasma Phys. Control. Fusion, 58:114004, 2016.

[19] R.B. White et al. Phys. Fluids, 26:2958, 1983.

[20] M. Garcia-Munoz et al. Phys. Rev. Lett., 104:185002, 2010.

[21] D. Pace et al. Plasma Phys. Control. Fusion, 53:062001, 2011.

[22] L-G. Eriksson, T. Hellsten, and U. Willen. Nucl. Fusion, 33:1037, 1993.

[23] M. Mantsinen et al. Analysis of icrf heating and icrfdriven fast ions in recent jet experiments. Presented at 
14th IAEA Technical Meeting on Energetic Particles in Magnetic Confinement Systems, Vienna 2015, 2015.

[24] G.D. Kerbel and M.G. McCoy. Phys. Fuids, 28:3629, 1985.
[25] E. Hirvijoki et al. Comput. Phys. Commun., 185:1310, 2014. 